\title{
A 19-Channel d.c. SQUID Magnetometer System for Brain Research
}

J. FlOKSTRA, H. J. M. TER BRAKE, E. P. HOUWMAN, D. VELDHUIS, W. JASZCZUK, M. CASPARI and H. ROGALLA University of Twente, Faculty of Applied Physics, P.O. Box 217, 7500 AE Enschede (The Netherlands)

A. MARTÍNEZ

Universidad de Zaragoza, E.T.S.I.I., Maria Zambrano 50, 50015 Zaragoza (Spain)

C. RILLO

I.C.M.A., C.S.I.C.-Universidad de Zaragoza, Servicio de Instrumentacion Cientifica, 50009 Zaragoza (Spain)

\section{Abstract}

A 19-channel d.c. SQUID magnetometer system for neuromagnetic investigations is under constuction. The first-order gradiometers for sensing the signal are placed in a hexagonal configuration. D.c. SQUIDs based on niobium/aluminium technology have been developed, leading to a field sensitivity of about $5 \mathrm{fT} / \sqrt{ } / \mathrm{Hz}$. SQUID read-out is realized with a resonant transformer circuit at $100 \mathrm{kHz}$. The multichannel control and detection electronics are compactly built.

\section{Introduction}

The superconducting quantum interference device (SQUID) is currently the most sensitive detector for measuring magnetic flux. SQUIDs are used in a large variety of applications of which neuromagnetism is the most remarkable [1]. The very weak fields originating from neural activity, being generally of the order of 10 to $1000 \mathrm{fT} / \sqrt{ } / \mathrm{Hz}$ in the frequency range from d.c. up to about $100 \mathrm{~Hz}$ [2], can be detected by a SQUID sensor. The d.c. SQUID has an intrinsic flux sensitivity of about $10^{-5}$ to $10^{-6} \phi_{0} / \sqrt{ } / \mathrm{Hz}$ ( $\phi_{0}$ is the flux quantum, $2.07 \times 10^{-15} \mathrm{~Wb}$ ), leading to a field sensitivity below $10 \mathrm{fT} / \sqrt{\mathrm{Hz}}$. The first magneto encephalograms (MEG) were measured point by point, using the cryogenic equipment at a regular grid of measurement points around the head in order to map the brain field distribution. For obtaining an acceptable signal-to-noise ratio, signal averaging has to be performed in many cases, so that in general only brain activity evoked by repeatable stimuli can be measured. This method is rather time consuming and often very inconvenient for the person under investigation. Further, it is not possible to measure adequately single brain events, like epilepsy. Nowadays, multichannel SQUID magnetometers have been developed by industrial and university groups in order to overcome the above-mentioned problems.

In this paper we describe several aspects of the development of a 19-channel d.c. SQUID magnetometer system that will be used in the $\mu$-metal shielded room of the Biomagnetic Centre Twente. Attention will be paid to the d.c. SQUID sensor including the pick-up coil configuration (Section 2) and the multichannel electronics system (Section 3).

\section{D.c. SQUID Sensor}

The d.c. SQUID is in principle a superconducting loop interrupted by two Josephson junctions. The flux-voltage transfer function of the sensor is sinusoidal if an adequate bias current is applied and the sensitivity is of the order of $10-100 \mu \mathrm{V} / \phi_{0}$ at the maximum slope. The SQUID is generally made in planar technology and flux is coupled to the SQUID ring by means of a planar multiturn input coil on top of the washer-like configu-

(C) Elsevier Sequoia/Printed in The Netherlands 


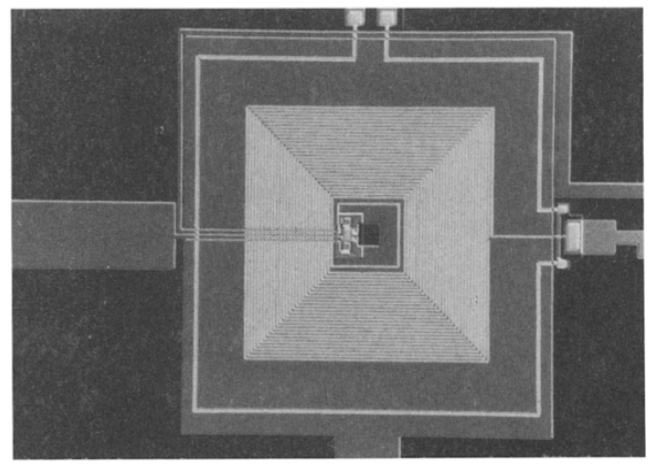

Fig. 1. Standard-type d.c. SQUID. The modulation coil ( 1 turn) and the input coil are on top of the washer. Typical outer dimensions $1 \mathrm{~mm} \times 1 \mathrm{~mm}$.

ration. As an illustration one of our SQUIDs is shown in Fig. 1.

As a first step to SQUID fabrication, a reliable and well-controlled technique was developed for preparing high-quality Josephson tunnel junctions on the basis of niobium/aluminium technology [3]. Trilayers of $\mathrm{Nb} / \mathrm{Al}$, $\mathrm{AlOx} / \mathrm{Nb}$ were prepared by d.c. magnetron sputter deposition, the aluminium oxide barrier being obtained by thermal oxidation of the aluminium at low oxygen pressure at room temperature. The junction areas of typically $5 \mu \mathrm{m} \times 5 \mu \mathrm{m}$ were defined by anodization of the trilayers protecting the junction area with photoresist. The junctions typically have gap voltages of $2.8-2.9 \mathrm{mV}$, critical currents of about $75 \%$ of the theoretical value and high sub-gap resistances leading to values of the quality parameter $V_{\mathrm{m}}$ of about $70 \mathrm{mV}$ at $4.2 \mathrm{~K}$ and $1 \mathrm{~V}$ at $1.2 \mathrm{~K}$. This demonstrates the high quality of our process.

Several SQUID configurations have been fabricated [4] using the technology developed for the Josephson junctions. The junctions are shunted by $\mathrm{Pd}$ resistances of about 3$5 \Omega$. The standard-configuration SQUIDs (Fig. 1) have been made with hole dimensions of $70 \mu \mathrm{m} \times 70 \mu \mathrm{m}$ and $145 \mu \mathrm{m} \times$ $145 \mu \mathrm{m}$. The junctions are placed at the inner side of the washer. The number of turns of the input coil is between 20 and 30 . The peak-to-peak value of the flux-voltage transfer function is found to be about $30 \mu \mathrm{V}$ being in agreement with our calculations. The flux

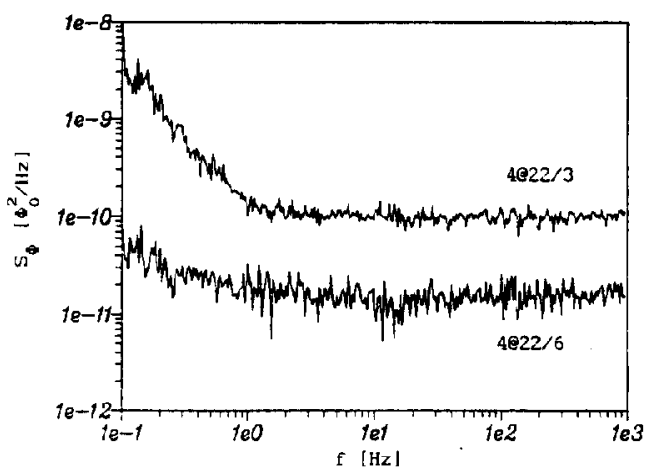

Fig. 2. Noise spectra of two standard d.c. SQUIDs. 4 (a) 22/3: SQUID hole, $145 \mu \mathrm{m} \times 145 \mu \mathrm{m}$; equivalent field noise in sensing coil, $5 \mathrm{fT} / \sqrt{ } / \mathrm{Hz} .4(a) 22 / 6$ : SQUID hole, $70 \mu \mathrm{m} \times 70 \mu \mathrm{m}$; equivalent field noise in sensing coil, $3.5 \mathrm{fT} / \sqrt{\mathrm{Hz}}$.

noise according to theory is about $2 \times 10^{-6} \phi_{0} / \sqrt{ } \mathrm{Hz}$, but the experimental values are a factor of five to eight higher. This additional noise is most probably caused by resonances due to coupling between SQUID and input coil. The $1 / f$-noise corner point is about $1 \mathrm{~Hz}$. Flux noise spectra for two of these SQUIDs are shown in Fig. 2.

Apart from the standard-type SQUID, resistively and inductively shunted SQUIDs were also fabricated. The SQUID hole is shunted by a resistance equal to the junction shunt resistance or an additional large-area loop, respectively. In these types of SQUIDs an input coil with a fixed inductance can be realized with a smaller number of turns as compared to the standard type, so that the deteriorating effect of the resonances can be diminished. The theoretical noise is about $1.5 \times 10^{-6} \phi_{0} / \sqrt{ } \mathrm{Hz}$, whereas the experimental values appeared to be only a factor of two to three higher.

The SQUID is connected to the pick-up coil section for sensing the magnetic fields. The pick-up coils consist of wire-wound firstorder gradiometers having two sections of three turns with a diameter of $20 \mathrm{~mm}$ and a base line length of $40 \mathrm{~mm}$ [5]. The distance between the turns of the lower sensing section is $0.5 \mathrm{~mm}$, whereas that of the turns of the compensating section is $6 \mathrm{~mm}$. The self inductance of the pick-up coil is $0.8 \mu \mathrm{H}$ and that of the input coil of the SQUID about $0.1 \mu \mathrm{H}$. 
Our magnetometer has 19 channels placed in a hexagonal configuration somewhat tilted with respect to each other ( 9.5 degrees) in order to fit to the concavely shaped bottom of the tail of the cryostat. In addition, three channels are available for measuring the orthogonal field components in order to compensate the imbalance effects of the gradiometers. Furthermore two channels are present to measure two spatial derivatives for further noise rejection. The flux transformer circuits in a multichannel system may easily invoke crosstalk and in order to eliminate this, external feedback to the flux transformer is applied [6].

The SQUID chip is placed in a rigid, compartmentalized $\mathrm{Nb}$ module. The module, consisting of several shielded sections, also contains the feedback inductor and the resonating circuit for the SQUID read-out.

\section{Electronics}

The read-out of the SQUID is realized by using this periodic flux -voltage converter in a flux-locked loop. A modulation signal with a frequency of $100 \mathrm{kHz}$ and an amplitude equivalent to $\phi_{0} / 4$ is applied to the SQUID for biasing at maximum sensitivity. Feedback from the output of the detection electronics to the SQUID gives the linearization of the magnetometer in the frequency range of interest $(<1 \mathrm{kHz})$.

The block diagram of a single channel of the electronics system is shown in Fig. 3. The dashed box contains all channel elements placed inside the cryostat. The preamplifiers are inside an r.f. shielded aluminium box internally compartmentalized and placed on top of the cryostat. The other elements of the Figure are outside the magnetically shielded room in a separate r.f. shielded cabinet.

The preamplifier consists of U311 FETs in a cascode configuration, loaded with an $R L C$ network resonating at the modulation frequency of $100 \mathrm{kHz}$. The preamplifier voltage noise $E_{\mathrm{n}}$ is about $1 \mathrm{nV} / \sqrt{\mathrm{Hz}}$ at this frequency. The quality factor is about five, thus giving a bandwidth of $20 \mathrm{kHz}$. The gain is about 50, being enough to make the noise contributions of the following stages negligible compared to the preamplifier noise.

A small resonant transformer is used between the SQUID and the preamplifier in order to reduce the effect of the preamplifier noise with respect to the SQUID noise. The forward transfer ratio of the SQUID is

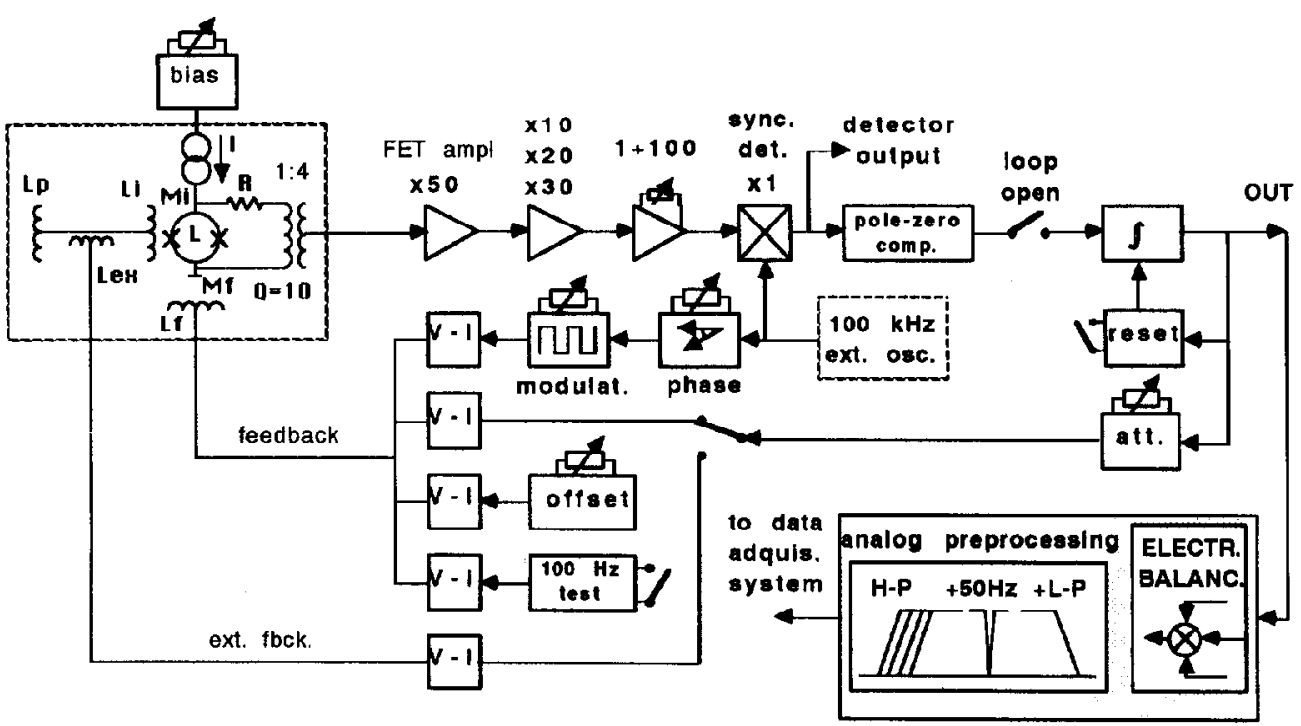

Fig. 3. D.c. SQUID control and detection electronics and analog preprocessing. 
roughly $70 \mu \mathrm{V} / \phi_{0}$ so that a minimum gain of about 20 is needed for the resonant transformer in order to limit the electronic noise contribution to about $10^{-6} \phi_{0} / \sqrt{\mathrm{Hz}}$. The current noise $I_{\mathrm{n}}$, being about $40 \mathrm{fA} / \sqrt{\mathrm{Hz}}$, is dominated by the impedance of the transfer line between the preamplifier and resonant transformer, and it gives a negligible contribution to the total noise. The selected gain for the transformer is 40 . A moderate $Q$-factor of 10 was chosen to give a bandwidth of $10 \mathrm{kHz}$, so that the voltage gain of the transformer should be about four. A prototype transformer has been made of $0.1 \mathrm{~mm} \mathrm{Cu}$ wire. It is a cylindrical coil former with a length of $5 \mathrm{~mm}$ and an outer diameter of $5.8 \mathrm{~mm}$, thus fitting in the $7 \mathrm{~mm}$ hole of the SQUID module. The prototype consisted of 90 primary and 635 secondary turns. The measured inductances are $17.4 \mu \mathrm{H}(27.5 \mu \mathrm{H})$ and $490 \mu \mathrm{H}(760 \mu \mathrm{H})$ respectively, whereas the coupling factor appeared to be $0.81(0.89)$ (transformer voltage gain $4.3(4.7)$ ). The values between brackets were measured outside the superconducting enclosure. The series resistances of the coils are below $1 \Omega$ so that the noise contribution can be neglected. The transformer is brought into resonance at the modulation frequency of $100 \mathrm{kHz}$ with a shunt capacitor connected at the secondary side and placed inside the SQUID module.

The detector/controller electronics are realized in a miniaturized version on a single Eurocard for each channel. Because of the miniaturization and the fact that the system is not designed to be a general purpose magnetometer, several parameters have been fixed, including the sensitivity and the frequency bandwidth $(5 \mathrm{kHz})$. The sensitivity is $1 \mathrm{~V}$ at the output for $1 \phi_{0}$ in the SQUID. The full scale of $10 \mathrm{~V}$ corresponds to an effective field of about $3 \mathrm{nT}$ in the gradiometer. The expected dynamic range in a $100 \mathrm{~Hz}$ measurement bandwidth is $2.5 \times 10^{5}$. Both a step-gain and a variable-gain amplifier for adjusting the open-loop gain are placed between the preamplifier and the phase-sensitive detector. An external oscillator provides the modulation signal for all channels. The flux modula- tion phase and peak-to-peak amplitude are set by potentiometers in the detector/controller unit.

The open-loop transfer function has a lowfrequency pole of the integrator, and two high-frequency poles due to the resonant transformer $(5 \mathrm{kHz})$ and the $R L C$ load of the preamplifier $(10 \mathrm{kHz})$. Furthermore, a laglead pole-zero compensation circuit has been added for dynamic behaviour optimization [7]. The slew rate is $7500 \phi_{0} / \mathrm{s}$ after compensation.

The electronics have been developed independently from the d.c. SQUID devices. System parameters such as dynamic behaviour, the gain of the feedback path and the openloop gain have been adjusted by means of a d.c. SQUID simulator [8].

Other modules included in the electronics are a manual/automatic reset, an offset for zcro adjustment, and a $100 \mathrm{~Hz}$ saw-tooth generator for optimum bias current, modulation amplitude and phase-shift settings. A switch selects between internal or external feedback. The sensitivity is adjusted with an attenuator in the feedback path.

Before the $A / D$ conversion the signal is first processed with the electronic balancing module [9] and then some analog filtering is necessary. For this purpose we developed a $50 \mathrm{~Hz}$ adaptive notch filter that suppresses the mains frequency by more than $60 \mathrm{~dB}$ with a bandwidth of only $1 \mathrm{~Hz}$. Further, high-pass and low-pass filters will be applied, the latter also functioning as anti-alias filters for the $1 \mathrm{kHz}$ sampling frequency. Thus signals can be measured without significant phase shift up to a frequency of $100 \mathrm{~Hz}$, which is adequate for most biomagnetic experiments.

\section{Conclusions}

A 19-channel d.c. SQUID magnetometer is under construction. A reliable and wellcontrolled technique for the fabrication of SQUID sensors based on $\mathrm{Nb}$ and $\mathrm{Al}$ has been developed. SQUIDs with a noise level between $5 \times 10^{-6}$ and $10^{-5} \phi_{0} / \sqrt{ } \mathrm{Hz}$ have been 
fabricated so that magnetic fields below $10 \mathrm{fT} / \sqrt{ } \mathrm{Hz}$ can be measured. Miniaturized multichannel control and detection electronics have been developed.

\section{References}

1 J. Clarke, SQUIDs, brains and gravity waves, Phys. Today, (1986) 36-44.

2 G. L. Romani, Biomagnetism: An application of SQUID sensors to medicine and physiology, Physica $B, 126$ (1984) 70-81.

3 E. P. Houwman, D. Veldhuis, J. Flokstra and H. Rogalla, Fabrication and properties of Nb/Al, Alox/ $\mathrm{Nb}$ Josephson tunnel junctions with a double oxide barrier, J. Appl. Phys., 67 (1990) 1992-1994.

4 E. P. Houwman, D. Veldhuis, J. Flokstra and H. Rogalla, Comparison of shunted DC-SQUIDs with large $\beta$, Physica B, 165/166 (1990) 81-82.
5 H. J. M. ter Brake, J. Flokstra, E. P. Houwman, D. Veldhuis, W. Jaszczuk, A. Martínez and H. Rogalla, Design and construction of a 19-channel DC-SQUID neuromagnetometer, Physica B, 165/166 (1990) 9596.

6 H. J. M. ter Brake, F. M. Fleuren, J. A. Ulfman and J. Flokstra, Elimination of flux-transformer crosstalk in multichannel SQUID magnetometers, Cryogenics, 26 (1986) 667-670.

7 C. Rillo, D. Veldhuis and J. Flokstra, Optimization of the dynamic behavior of a SQUID system using an electronic simulation, IEEE Trans. Instrum. Meas., IM-36 (1987) 770-775.

8 A. Martínez, J. Flokstra, C. Rillo, L. A. Angurel, L. M. Garcia and H. J. M. ter Brake, Low noise SQUID simulator with large dynamic range of up to eight flux quanta, Cryogenics, 30 (1990) 324 329.

9 H. J. M. ter Brake, Z. Dunajski, W. A. G. van der Mheen and J. Flokstra, Electronic balancing of multichannel SQUID magnetometers, J. Phys. E: Sci. Instrum., 22 (1989) 560-564. 